\title{
Martin A. Makary (eds): General Surgery Review (2nd Edition)
}

\author{
Ladner-Drysdale, Washington D.C., London, Singapore, 2008, Paperback, 575 pp, \\ \$74.99 (amazon.com), ISBN 0-9760662-2-X
}

\author{
Markus Schäfer
}

Published online: 16 January 2010

(C) Société Internationale de Chirurgie 2010

This book is the second edition of the kind of books that have become popular in recent years. Whereas "classic" surgical texts usually provide a comprehensive overview, they do not offer a rapid answer to clinical problems because of the extensive amount of data offered. In contrast, simple checklists provide information limited to the key issues but lack any background material.

The current edition of General Surgery Review fills the gap between the aforementioned types of books. It covers a very broad spectrum of topics on nearly all surgical subspecialties: anesthesia, trauma, critical care, and basic science. The most important and comprehensive chapter is dedicated to general and gastrointestinal surgery. The book ends with a brief, but very nice, chapter on medical statistics, patient safety, and medical ethics. Each topic within a chapter starts with a clinical situation and specific question. Whereas the answer is summarized in one or two sentences, background about it is provided, including all aspects of diagnosis, management, and outcome-but in a concise manner. Therefore, this book may be particularly helpful preparing clinical examinations for residents and medical students. Teachers and experts also may benefitin their daily practice-from the precise clinical questions and meticulous answers.

The 70 plus contributors are experts are from the United States-the majority is affiliated with the Johns Hopkins University School of Medicine. This obviously gives this book a super strong American flair. Like most such multiauthor books, the chapters are of different quality. This

\section{Schäfer $(\bowtie)$}

Department of Visceral Surgery, Centre Hospitalier

Universitaire Vaudois, University Hospital, Lausanne, Switzerland

e-mail: markus.schafer@chuv.ch second edition has been expanded, and new chapters have been added (i.e., cell biology, cytokines, and immunology). Nearly all topics of surgery are now covered, and this book can really be used as a single source of information. There are more pictures and diagrams included; therefore, visualization of surgical procedures is facilitated. A big advantage for teachers is the free online availability of illustrations for educational purposes.

There are some weak points, in particular for European or non-American readers. First, no SI units are used, e.g., lb instead of $\mathrm{kg}$. Second, interdisciplinary approaches to cancer patients should be better explained. Third, no operative techniques are provided for common problems, e.g., Lichtenstein technique for inguinal hernia repair. Fourth, it would be helpful, in particular for medical students and residents, if the authors could rank the relevance of the different topics within a chapter (e.g., frequent clinical problem, important oncologic issue). Finally, the quality of the book would significantly improve if the authors could provide an outlook on future developments within a given topic.

Overall, I like this book because it provides a thorough but concise overview of surgery. It can be recommended as a further source of information for residents and teachers who have to deal with clinical examinations. There are similar books in German and French, with a particular focus on local national board examinations for general surgery.

To conclude by John L. Cameron, who wrote in the preface, on the value of this book: "When the second edition of a text is published, it indicates that the first edition was a success and both the publisher and the editor wish it to continue. And even more importantly it means the readers thought it useful and of value." 